\title{
Gorham-Stout Disease Mimicking Spondyloarthritis
}

HYE SOO KOO, MD; SO YOUNG PARK, MD, Department of Radiology; GOU YOUNG KIM, MD, Department of Pathology; RAN SONG, MD, Division of Rheumatology, Department of Internal Medicine, Kyung Hee University Hospital at Gangdong, School of Medicine, Kyung Hee University, Seoul, Republic of Korea. Address correspondence to Dr. S.Y. Park, Department of Radiology, Kyung Hee University Hospital at Gangdong, 892, Dongnam-ro, Gangdong-gu, Seoul 134-727, Republic of Korea. E-mail: balgundol@hanmail.net. J Rheumatol 2014;41:1430-2; doi:10.3899/jrheum.131439

Gorham-Stout disease (GSD) is a rare disorder characterized by the proliferation of non-neoplastic thin-walled vascular tissue associated with progressive osteolysis ${ }^{1}$.

A 37-year-old woman with a 7-year history of chronic bilateral pelvic pain was evaluated for aggravated left hip pain. She was diagnosed with spondyloarthritis (SpA) 4 years prior and was receiving medical treatment. Laboratory analyses were normal. Radiography and computed tomography (CT) of the pelvis showed widening of both sacroiliac joints and multifocal osteolytic lesions in the bony pelvis (Figure 1A-B, arrows). Because a diagnosis of GSD was considered, incisional biopsies of the right ilium and surrounding soft tissue were performed. Histologic analysis revealed thin-walled, blood-filled vascular cavities and hypervascular fibrous connective tissue replacing the cortex and medulla of the right ilium (Figure 1C), and a focal vascular proliferation in the surrounding skeletal muscle. GSD was diagnosed on the basis of radiographic appearance and histopathological features. The patient had been treated for years under a misdiagnosis of SpA. She was treated by antiosteoclastic medication (alendronate) as well as conservative management without progression.
GSD is a rare disorder of unknown etiology characterized by the proliferation of vascular channels causing osteolysis. The clinical presentation is variable depending on the site of involvement ${ }^{1}$. Multiple osteolytic lesions can be seen on $\mathrm{CT}$, as can lesions with altered signal change and enhancement on magnetic resonance imaging ${ }^{2,3}$. Soft-tissue involvement and splenic lesions are also common, and chylothorax may occur ${ }^{2}$. The treatment for GSD includes antiosteoclastic medications, interferon $\alpha-2 b$, radiation therapy, and surgical reconstruction or stabilization ${ }^{1}$.

\section{REFERENCES}

1. Patel DV. Gorham's disease or massive osteolysis. Clin Med Res 2005;3:65-74.

2. Kotecha R, Mascarenhas L, Jackson HA, Venkatramani R. Radiological features of Gorham's disease. Clin Radiol 2012;67:782-8.

3. Manisali M, Ozaksoy D. Gorham disease: correlation of MR findings with histopathologic changes. Eur Radiol 1998;8:1647-50. 

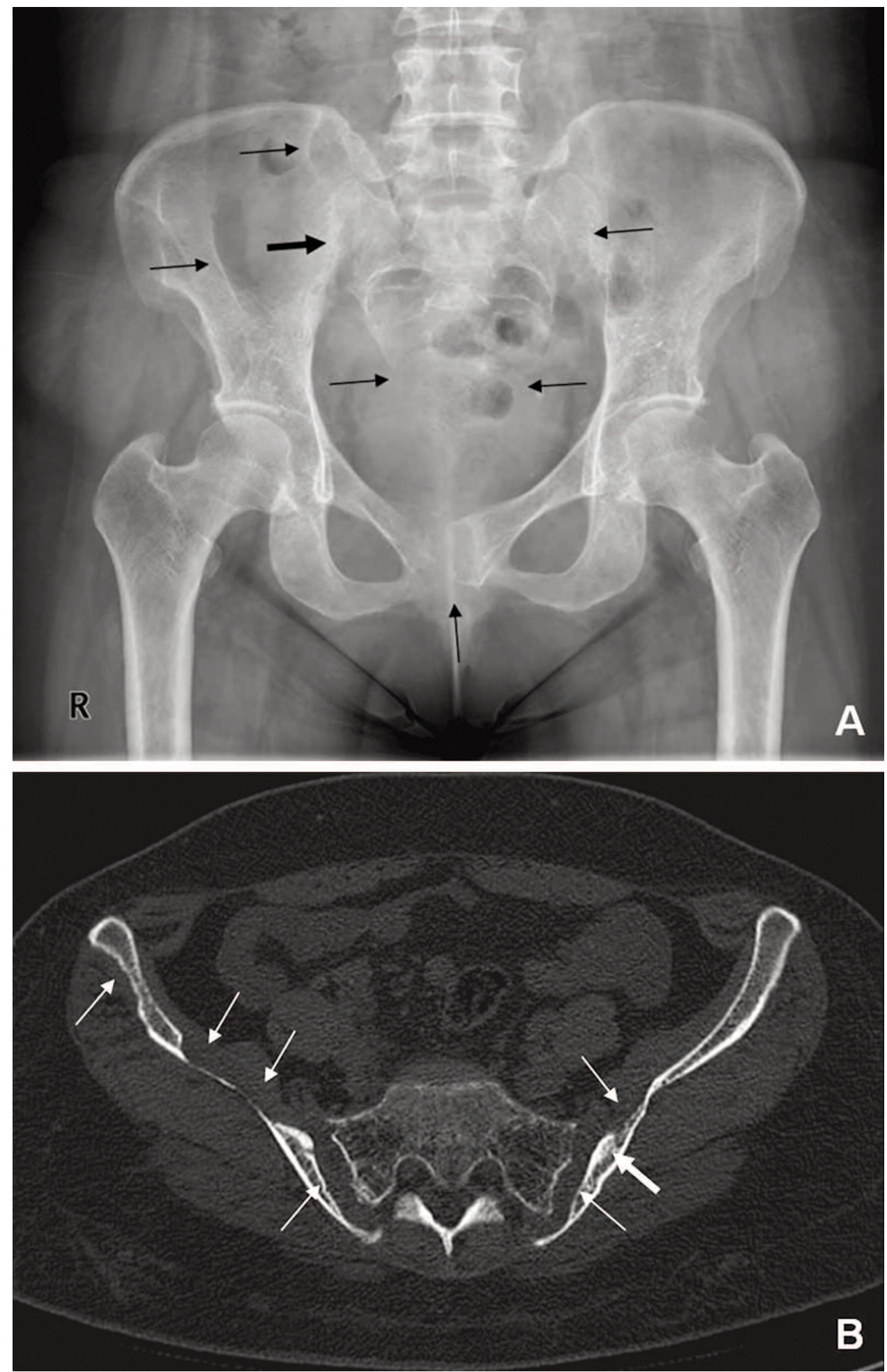

Figure 1. A. Radiography of the pelvis showed diffuse widening of both sacroiliac joints and osteolytic areas in the right ilium, symphysis pubis, lower sacrum, and coccyges (arrows). Sclerosis is seen at ilial side of right sacroiliac joint (thick arrow). B. Computed tomography of the pelvis revealed widening of both sacroiliac joints and erosion-like osteolytic areas in both ilii (arrows). Focal sclerosis at ilial side of left sacroiliac joint (thick arrow) is seen. 


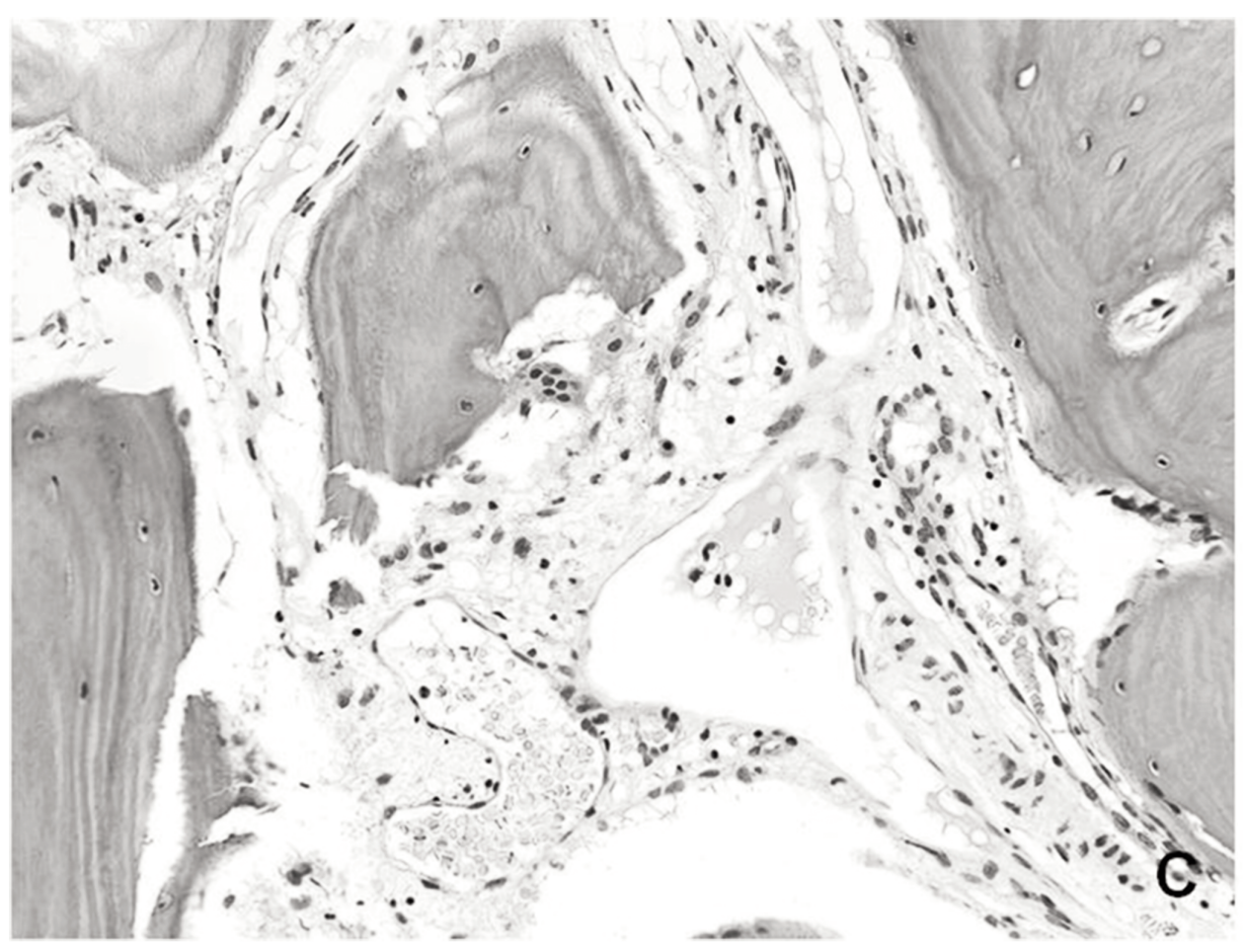

Figure 1. C. Microscopically, abnormal vascular proliferation with some dilated vessels in fibrous bone marrow space is noted (H\&E stain, original magnification $\times 200)$. 